\title{
Smectic nanostructures with a typical size less than a visible light wavelength: physics and electro-optics
}

\author{
E. P. Pozhidaev, ${ }^{1,2}$ V. E. Molkin, ${ }^{2}$ V. G. Chigrinov ${ }^{1}$ \\ ${ }^{1}$ Hong Kong University of Science and Technology, Clear Water Bay, Kowloon, Hong Kong \\ ${ }^{2}$ P.N. Lebedev Physical Institute of Russian Academy of Sciences, Leninsky pr. 53, Moscow, 19991, Russia
}

Received February 15, 2011; accepted March 28, 2011; published March 31, 2011

\begin{abstract}
Very short helix pitch $\left(\mathrm{p}_{0} \cong 150-200 \mathrm{~nm}\right)$ ferroelectric liquid crystals (FLCs) have been developed. Electro-optical cells based on these mixtures are free from selective reflection and diffraction in the visible spectral range and therefore exhibit very perfect operation in deformed helix ferroelectric (DHF) mode. Two main electro-optical modes of DHF cells with sub-wavelength helix pitch are under consideration
\end{abstract}

Deformed helix ferroelectric liquid crystal cells (DHFLC) $[1,2]$ could be principally applicable in displays, optical communication systems and photonic devices [3-6] as the fastest electro-optical mode of liquid crystals in a broad temperature range [6, 7]. The electrically controlled birefringence $\Delta n_{\text {eff }}(E)=<\mathrm{n}_{\mathrm{e}}(\mathrm{E})-\mathrm{n}_{\mathrm{o}}(\mathrm{E})>$ is a sequence of the FLC helical structure deformation and deviation $\Delta \alpha(E)$ of the FLC principle axis in electric field $[1,2,9,10]$, see Fig. 1.
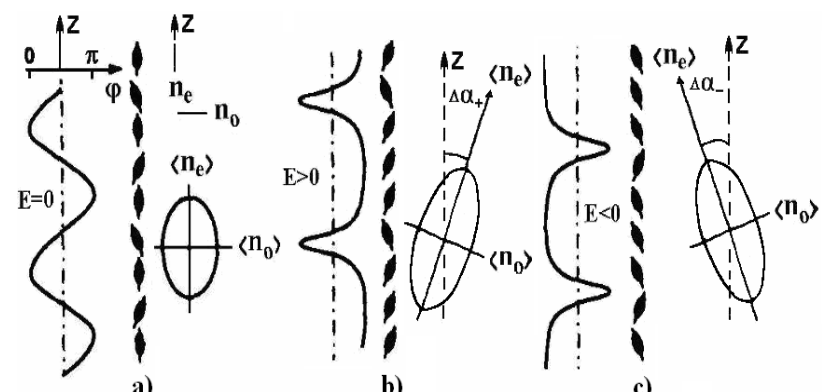

Fig. 1. Left is azimuthal $\varphi(z)$ angle distribution, in the center local distribution of the refractive index ellipsoid along $z$ axis, which corresponds to the rubbing direction, right averaged over the light aperture refractive index ellipsoid: a) $\mathrm{E}=0$; b) $\mathrm{E}>0$, c) $\mathrm{E}<0$.

At sub-wavelength helix pitch, the light transmission $T_{h}$ of the DHFLC placed between crossed polarizers is expressed as [9-11]:

$$
T_{h}=\sin ^{2} 2[\beta \pm \Delta \alpha(E)] \cdot \sin ^{2} \frac{\Delta \Phi(E)}{2},
$$

where $\beta$ is the angle between the input linear polarized light polarizing plane and $\mathrm{z}$ axes (see Figure 1), and $\Delta \Phi$ is written as:

$$
\Delta \Phi(E)=\frac{2 \pi d_{F L C} \Delta n_{e f f}}{\lambda}
$$

Here, $\lambda$ is the wavelength, $d_{F L C}$ is the FLC layer thickness, and $\Delta n_{\text {eff }}=\Delta n_{\text {eff }}^{E=0}+\Delta n_{\text {eff }}(E)$.

If the incident polarized light propagates parallel to smectic layers of the DHFLC with a sub-wavelength helix pitch then, according to $[9,10]$ the electrically controlled birefringence is proportional to electric field $E$ square:

$$
\Delta n_{e f f}(E)=\left(n_{e}-n_{0}\right) \frac{\sin ^{2} 2 \theta}{1-\frac{3}{2} \sin ^{2} \theta} G^{2} E^{2}
$$

Here $\theta$ is the tilt angle, $n_{0}, n_{e}$ are the ordinary and extraordinary refractive indices, correspondingly, and

$$
G=\frac{\varepsilon_{0} \chi_{G}}{P_{s}}
$$

where $\varepsilon_{0}$ is the vacuum permittivity, $P_{s}$ is the spontaneous polarization; $\chi_{G}$ is the Goldstone mode dielectric susceptibility. Deviation $\Delta \alpha(E)<<\theta$ of the FLC principle axis in electric field at $\beta=0$ is proportional to electric field [10]:

$$
\Delta \alpha(E)=\frac{\sin 2 \theta}{2\left(1-\frac{3}{2} \sin ^{2} \theta\right)} G E
$$

So, both $\Delta n_{\text {eff }}(E)$ and $\Delta \alpha(E)$ depend on dielectric susceptibility $\chi_{G}$ while its well known inherent property is dielectric dispersion. Hence, $\Delta n_{\text {eff }}$ and $\Delta \alpha$ should be frequency dependent as well.

Typical dielectric dispersion of DHFLC with a sub-wavelength helix pitch is observed very clearly in a broad frequency range between $1 \mathrm{kHz}$ and $100 \mathrm{kHz}$, see Fig. 2. DHF FLC-587, which was elaborated by the author, has been used in experiments. The main parameters of the FLC-587 at $\mathrm{T}=22^{\circ} \mathrm{C}$ are listed below: spontaneous polarization $P_{s}=1.5 \cdot 10^{-3} \mathrm{C} / \mathrm{m}^{2}$, the helix pitch $p_{0}=150 \mathrm{~nm}$, the tilt angle $\theta=35^{\circ}$. 


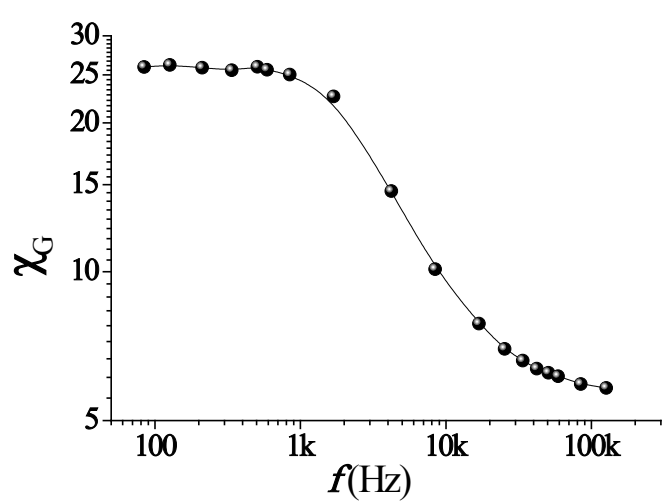

Fig. 2. Dielectric dispersion of the Goldstone mode dielectric susceptibility of DHF FLC-587, which was elaborated by the author.

Measurements done at the FLC layer thickness $3 \mu \mathrm{m}, \mathrm{T}=22^{\circ} \mathrm{C}$

Dispersions $\Delta n_{\text {eff }}(f)$ and $\Delta \alpha(f)$ have been measured at the same frequency range (see Fig. 3) as the dispersion $\chi_{G}(f)$ shown in Fig. 2. Dependencies $\Delta n_{\text {eff }}(f)$ and $\Delta \alpha(f)$ show, although without exact match, the same trends as it can be calculated substituting measured $\chi_{G}(f)$ data (see Fig. 2) to formulas (3)-(5).

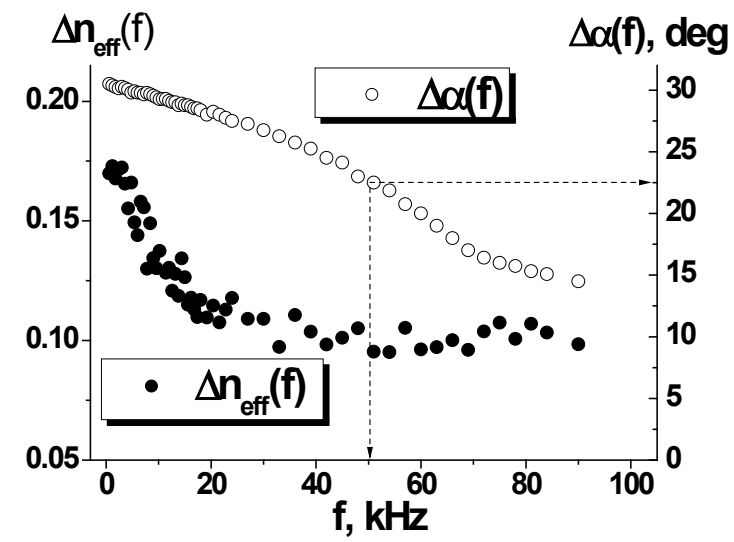

Fig. 3. Dependencies of $\Delta n_{\text {eff }}$ and $\Delta \alpha$ on the applied voltage frequency are presented. Measurements have been carried out at the FLC layer thickness $3 \mu \mathrm{m}, \mathrm{T}=22^{\circ} \mathrm{C}, \lambda=0.628 \mu \mathrm{m}$.

Two main electro-optical effects are observed in DHFLC. The difference between these effects is associated primarily with the incident light polarization plane orientation relative to the principle optical axis of the helical structure. Two orientations that correspond to two main electro-optical modes of DHFLC are the most important:

$$
\begin{aligned}
& |\beta|=|\Delta a|, \\
& \beta=0 \text { or } \pi / 2
\end{aligned}
$$

Under the condition (6) the electro-optical response is sensitive to the polarity of the applied voltage (Fig. 4), similar to the response of SSFLC cells [12]. However, the response of the DHFLC cell (the response time $\tau_{0.1-0.9} \cong 1 \mu \mathrm{s}$ at the driving voltage frequency $50 \mathrm{kHz}$, see the lower oscillogram in Fig. 4) is considerably faster in comparison with SSFLC cells.

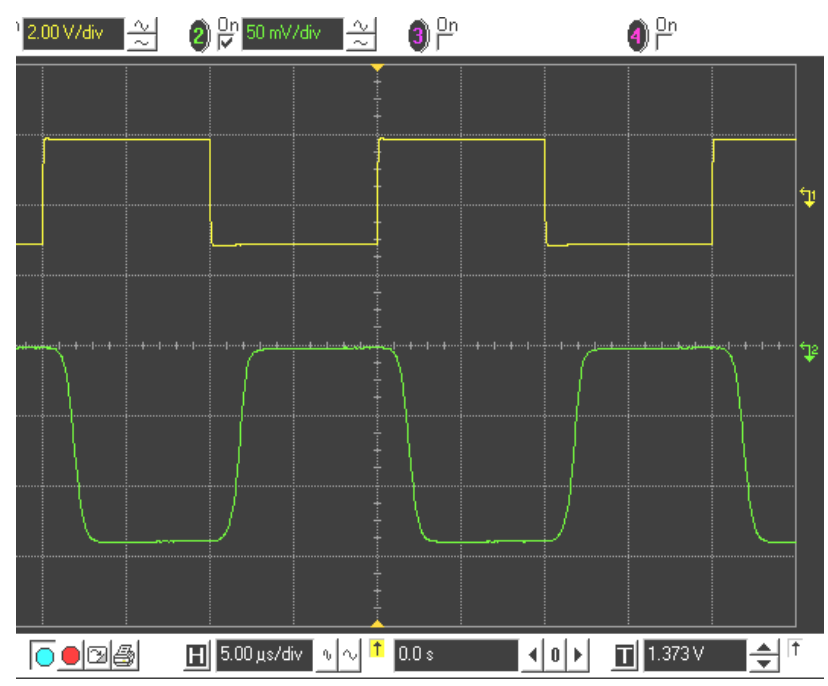

Fig. 4. A copy of an oscilloscope screen is presented here. The upper oscillogram shows the driving voltage waveform (whose amplitude is \pm 15 Volt and frequency is $50 \mathrm{kHz}$ ) applied to the FLC- 587 based cell with the FLC layer thickness $3 \mu \mathrm{m}$. The lower waveform shows the electro-optical response of the cell at $\beta=\Delta \alpha=22.5^{\circ}$, the frequency is $50 \mathrm{kHz}, \mathrm{T}=22^{\circ} \mathrm{C}, \lambda=0.628 \mu \mathrm{m}$

The reason for this enhancement is just the operation of the cell in the dispersion region (Fig. 3), where real deviations of molecules from their equilibrium positions are becoming smaller with applied voltage frequency increased. Consequently, the time needed to reach the new equilibrium positions, is also decreasing. But the point is that the condition to achieve maximum light transmission of the DHFLC is in no way connected with the magnitude of the deviations of the molecules. The main thing is, anyway, to satisfy the conditions of maximum light transmission:

$$
\begin{aligned}
& \beta=\Delta \alpha=22.5^{\circ}, \\
& \frac{\Delta n_{e f f} d_{F L C}}{\lambda}=\frac{1}{2},
\end{aligned}
$$

which arise from Eq. (1) and Eq. (2). For the cell which is under consideration, the conditions (8) and (9) are satisfied at the control voltage frequency $f=50 \mathrm{kHz}$, as shown by dotted arrows in Fig. 3. The conditions (8), (9) can be satisfied even at higher frequency than before; for example, at $f=85 \mathrm{kHz}$ [6] and when the electro-optical response time was $\tau_{0.1-0.9}=850 \mathrm{~ns}$. So, the electro-optical 
mode of DHFLC under the condition (6) provides very sub-microsecond response time, which is practically independent of temperature, as it was shown before [8].

Under the condition (7) the electro-optical response is insensitive to the driving voltage polarity (Fig. 5a), similar to nematic liquid crystal (NLC) cells, but the response time is two orders faster as compared with NLCs: $\tau_{0.1-0.9}^{\text {on }} \cong 80 \mu s, \tau_{0.1-0.9}^{\text {off }} \cong 60 \mu s$.

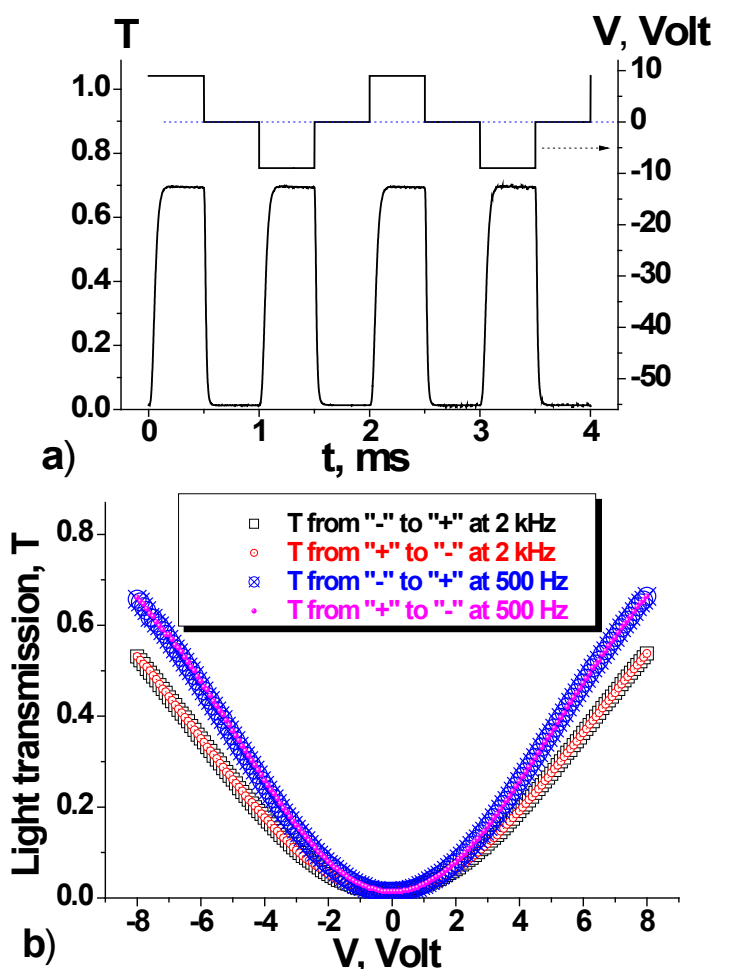

Fig. 5. a) top is the applied voltage; bottom is the electro-optical response of $1.7 \mu \mathrm{m}$ FLC-587 based cell, placed between crossed polarizers at $\beta=0$; b) V-shaped mode in the saturation levels of light transmission levels measured at rectangular alternating voltage, the response frequency is $f_{e l}=2 \mathrm{kHz}$ and $500 \mathrm{~Hz}$. The light transmission $T$ evaluated in comparison with transmission of empty cell placed between parallel polarizers. Solid black and dotted red lines are calculated transmissions at $f_{e l}=500 \mathrm{~Hz}$ and $2 \mathrm{kHz}$, respectively.
The response exhibits a perfect $\mathrm{V}$-shaped mode (Fig. $5 b)$ in the saturation levels of light transmission obtained for the case of rectangular alternating applied voltage pulses shown in Fig. 5a. As illustrated in Fig. 5a, the electro-optical response frequency $f_{e l}$ is two times higher than the driving voltage frequency $f_{a v}$. The reason of the hysteretic-free response (Fig. 5 b) is that the voltage waveform creates the conditions when hysteresis cannot be observed, since the addressing of light transmission levels starts from the same non-deformed helix that formed at zero voltage between two pulses (Fig. 5a).

So, the V-shaped electro-optical response is shown to be an inherent property of any DHFLC under a special choice of the applied rectangular alternating electric field waveform, frequency, and the cell geometry.

The support of HKUST grants CERG 612208, CERG 612310, CERG 612409, Russian Foundation of Basic Research, Grants 10-02-01336-a, 10-03-13305-PT-omi, 10-03-90016-Bel-a, Russian Federal Agency of Science and Innovations (project 02.740.11.5166) is gratefully acknowledged.

\section{References}

[1] L.A. Beresnev, L.M. Blinov, D.I. Dergachev, C.B. Kondratev. JETP Lett. 46(8), 328 (1987)

[2] L.A. Beresnev, V.G. Chigrinov, D.I. Dergachev, E.P. Pozhidaev, J. Funfshilling, M. Schadt, Liq. Cryst. 5(4), 1171 (1989).

[3] E.P. Pozhidaev, Proc. SPIE 4511, 92 (2001).

[4] F. Podgornov, E. Pozhidaev, D. Ganzke, W. Haase, IEEE Proc. ISAF 2000, 00CH37076, 1005 (2000).

[5] W. Haase, F. Podgornov, E. Pozhidaev, Proc. SPIE, 4481, 17 (2002).

[6] V.G. Chigrinov, H.S. Kwok, E.P. Pozhidaev, T.Z. Lu. Proc. Second Asia-Pacific Polymer Fiber Optics Workshop, 149 (2003).

[7] W. Haase, A. Suvorova, I. Chernyaev, N. Kundikova, E. Pozhidaev, F. Podgornov, Integr. Ferroelectr. 87, 3 (2007).

[8] E.P. Pozhidaev, S.A. Pikin, D. Ganzke, S.A. Shevtchenko, W. Haase, Ferroelectr. 246(1-4), 1141 (2000)

[9] I. Abdulhalim, G. Moddel, Mol. Cryst. Liq. Cryst. 200, 79 (1991).

[10] E. Pozhidaev, S. Torgova, M. Minchenko, C.A.R. Yednak, A. Strigazzi, E. Miraldi, Liq. Cryst. 37(8), 1067 (2010).

[11] V.G. Chigrinov, Liquid Crystal Devices: Physics and Applications (Artech House, Boston, London 1999).

[12] N.A. Clark, S.T. Lagerwall, J. Appl. Phys. 36, 899 (1980). 